\title{
Strengthened connections between engrams encode specific memories
}

\author{
Ji-il Kim, Dong Il Choi \& Bong-Kiun Kaang ${ }^{*}$ \\ School of Biological Sciences, Seoul National University, Seoul 08826, Korea
}

In previous studies, memory storage was localized to engram cells distributed across the brain. While these studies have provided an individual cellular profile of engram cells, their synaptic connectivity, or whether they follow Hebbian mechanisms, remains uncertain. Therefore, our recent study investigated whether synapses between engram cells exhibit selectively enhanced structural and functional properties following memory formation. This was accomplished using a newly developed technique called "dual-eGRASP". We found that the number and size of spines on CA1 engram cells that receive inputs from CA3 engram cells were larger than at other synapses. We further observed that this enhanced connectivity correlated with induced memory strength. CA3 engram synapses exhibited increased release probability, while CA1 engram synapses produced enhanced postsynaptic responses. CA3 engram to CA1 engram projections showed strong occlusion of long-term potentiation. We demonstrated that the synaptic connectivity of CA3 to CA1 engram cells was strengthened following memory formation. Our results suggest that Hebbian plasticity occurs during memory formation among engram cells at the synapse level. [BMB Reports: Perspective 2018; 51(8): 369-370]

Memory resides in the engram, i.e. it is the physical substrate of memory. Specific populations of neurons activated during memory formation are known to be necessary and sufficient for memory storage and expression. Thus, it is widely accepted

*Corresponding author. E-mail: kaang@snu.ac.kr

https://doi.org/10.5483/BMBRep.2018.51.8.176

\section{Received 7 July 2018}

Keywords: Dendritic spine, Dual-eGRASP, Hippocampus, Memory engram, Synaptic plasticity

Abbreviations: AMPA, $\alpha$-amino-3-hydroxy-5-methyl-4-isoxazolepropionic acid; GRASP, green fluorescent protein reconstitution across synaptic partners

Perspective to: Choi et al. (2018) Interregional synaptic maps among engram cells underlie memory formation, Science, 360(6387):430435, doi: 10.1126/science.aas9204. that these specific neuronal populations comprise the engram at a neuronal level (Josselyn et al (2015) Nat Rev Neurosci 16, 521-534). However, as proposed by Donald O. Hebb, the substrate of memory might be the strengthening of synapses between co-activated neurons. However, memory engrams have only been studied at the neuronal level, not the synaptic level. This is due to technical limitations preventing the comparison of synapses on a single postsynaptic neuron based on their presynaptic population.

To address this issue, we modified the GRASP technique (Feinberg et al (2008) Neuron 57, 353-365). By introducing a series of mutations and a weakly interacting domain that facilitates reconstitution, we developed "dual-eGRASP" featuring enhanced and distinguishable cyan or yellow fluorescent signals. By applying dual-eGRASP to the CA3 and CA1 engram cells, we tested Hebb's pioneering hypothesis, often paraphrased as "fire together, wire together". We labeled CA3 engram to the CA1 engram (E-E) synapses with yellow GRASP signals on red fluorescently labeled dendrites. This was accomplished through the expression of yellow pre-eGRASP in CA3 engram cells, and post-eGRASP, together with membrane-targeted mScarlet-I, in the contralateral CA1 engram cells. In addition, we expressed post-eGRASP and membrane-targeted iRFP670 in a sparse CA1 neuronal population, while we expressed cyan pre-eGRASP in a sparse CA3 neuronal population, in order to compare E-E synapses with other synapses (non-engram to engram $(\mathrm{N}-\mathrm{E})$, engram to non-engram $(\mathrm{E}-\mathrm{N})$, and non-engram to non-engram $(\mathrm{N}-\mathrm{N})$ synapses) (Diagram 1 ).

We found that the density of the E-E synapses was significantly higher than that of the E-N synapses. The density of $\mathrm{N}-\mathrm{N}$ and N-E synapses did not show any significant differences. Moreover, we found that the E-E spine head diameter and synaptic spine volume were significantly greater than in the N-E synaptic spines. Again, no significant differences were found between the $\mathrm{N}-\mathrm{N}$ and E-N synapses. In addition, we investigated whether memory strength alters connectivity between engram cells, since it is known that the number of engram cells remains constant across different memory strengths (Morrison et al (2016) Neurobiol Learn Mem 135, 91-99). We adjusted the memory strength by applying electric shocks of different intensities. This revealed that the density and spine size of E-E synapses were both

ISSN: 1976-670X (electronic edition)

Copyright (c) 2018 by the The Korean Society for Biochemistry and Molecular Biology

(c) This is an open-access article distributed under the terms of the Creative Commons Attribution Non-Commercial License (http://creativecommons.org/licenses/by-nc/4.0) which permits unrestricted non-commercial use, distribution, and reproduction in any medium, provided the original work is properly cited. 
A

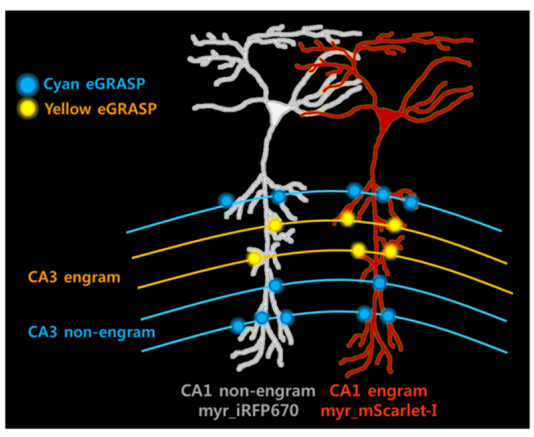

B
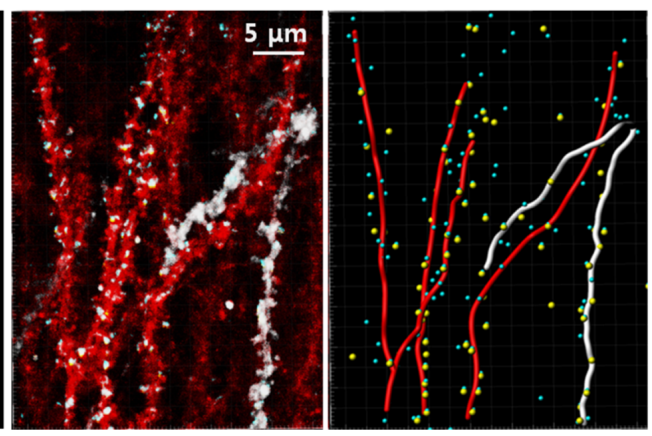

Diagram 1. Schematic diagram (A) and an example of 3D modeling image (B) of the four possible synapse types. Cyan circles representing cyan eGRASP signals indicate synapses originating from CA3 non-engram cells. Yellow circles representing yellow eGRASP signals indicate synapses originating from CA3 engram cells. CA1 non-engram and engram cells are shown in white and red, respectively. Adapted from Choi, et al., Science (2018). significantly greater in the strong shock group compared to those in the other groups. These results demonstrate that E-E synapses have greater structural connectivity than the other synaptic types investigated here. In addition, we showed that the magnitude of E-E synapse enhancement positively correlates with memory strength. We used electrophysiological experiments to further investigate how synaptic strength between engram cells changed. ChrimsonR and Chronos, which are independently activated using lasers of different wavelengths, were used to distinguish input from CA3 engram cells and total excitatory neurons, respectively. From this result, we found an increased release probability of CA3 engram inputs, and increased levels of postsynaptic AMPA receptors in CA1 engram cells. We also found complete pairing long-term potentiation occlusion in E-E synaptic responses. This may occur through the synergistic effects of increased presynaptic release probability and postsynaptic potentiation.

In summary, we developed dual-eGRASP, which classifies synapses on a single dendrite based on their presynaptic neuronal population in the rodent brain. It could contribute to more sophisticated connectome analyses when applied to multiple brain regions. Furthermore, our results revealed the increased structural and functional connectivity between CA3 engram and CA1 engram cells after memory formation.

\section{ACKNOWLEDGEMENTS}

This work was supported by the National Honor Scientist Program (NRF-2012R1A3A1050385) of Korea. 